\title{
Polynomial sPlines as eXamples of Chebyshevian SPLINES
}

\author{
M.-L. Mazure \\ Laboratoire Jean Kuntzmann \\ Université Joseph Fourier \\ BP53, 38041 Grenoble Cedex 9, France \\ mazure@imag.fr
}

The results we present here concern geometrically continuous polynomial splines, in the sense that the left/right derivatives at the knots are linked by connection matrices. A classical sufficient condition for spaces of such splines to be suitable for either Approximation or Geometric Design is the total positivity of all their connection matrices (i.e., all their minors are non-negative) [1]. Their entries can then serve as shape parameters. As an example, they can be used in spline interpolation to make up for the Gibbs phenomenon.

We show how to obtain all the connection matrices leading to suitable spline spaces. The results in themselves are interesting in so far as they permit more efficient shape effects than total positivity. Even more interesting is the way we achieve them. They follow from considering polynomial splines as special instances of Chebyshevian splines and polynomial spaces on a closed bounded interval as special instance of Extented Chebyshev spaces [3, 2].

\section{References}

[1] N. Dyn and C.A. Micchelli, Piecewise polynomial spaces and geometric continuity of curves, Numer. Math., 54 (1988), pp. 319-337.

[2] M.-L. Mazure, Finding all systems of weight functions associated with a given Extended Chebyshev space, J. Approx. Theory, 163 (2011), pp. 363-376.

[3] M.-L. Mazure, How to build all Chebyshevian spline spaces good for Geometric Design?, preprint. 